\title{
12 Jahre erfolgreiche Kooperation zwischen DVGW/DWA und der FH-DGGV
}

\author{
Roland Otto
}

Eingang des Beitrages: 10.6.2015 / Online veröffentlicht: 18.8.2015

(C) Springer-Verlag Berlin Heidelberg 2015

Interdisziplinäres Arbeiten hat heute einen hohen Stellenwert. Das trifft auch auf die wissenschaftlichen und praxisorientierten Bereiche $\mathrm{zu}$, in denen die Fachsektion Hydrogeologie (FH-DGGV) aktiv ist. So ist in der Geschäftsordnung im $\S 2$ Abs. 1 definiert:

Die Fachsektion Hydrogeologie hat den Zweck, die Kommunikation und Zusammenarbeit von Fachleuten verschiedener Disziplinen, die sich der Erforschung und Nutzanwendung des Grundwassers widmen, zu fördern. Die Fachsektion Hydrogeologie begreift sich als Forum für Wissenschaft und Praxis in der Hydrogeologie.

In diesem Zusammenhang ergeben sich zwangsläufig gemeinsame Themenfelder mit anderen Organisationen wie z. B. dem Deutschen Verein des Gas- und Wasserfaches e. V. (DVGW) oder der Deutschen Vereinigung für Wasserwirtschaft, Abwasser und Abfall e. V. (DWA). So hat sich der DVGW das Ziel gesetzt, „,das Gas- und Wasserfach in technischer und technisch-wissenschaftlicher Hinsicht unter besonderer Berücksichtigung der Sicherheit, des Umweltschutzes und der Hygiene zu fördern." Hierzu zählen u. a. die Ausarbeitung und Verbreitung des DVGW-Regelwerkes, die Sammlung, Ausweitung und Verbreitung von technischen und technisch-wissenschaftlichen Kenntnissen sowie die Anregung und Förderung technisch-wissenschaftlicher Vorhaben. Auch die DWA nennt in ihrer Satzung als Zweck, die Wasser- und Abfallwirtschaft zu fördern und die auf diesen Gebieten tätigen Fachleute zusammenzuführen. Hierbei wird explizit Wert auf die Zusammenarbeit mit

PD Dr. R. Otto $(\square)$

Landesamt für Landwirtschaft, Umwelt und ländliche Räume,

Hamburger Chaussee 25, 24220 Flintbek, Deutschland

E-Mail: roland.otto@1lur.landsh.de fachverwandten Vereinigungen, Einrichtungen und Organisationen im In- und Ausland gelegt.

Während DVGW und DWA ihren Schwerpunkt in der Wasser-, Abfall- und Energiewirtschaft sehen und dort ein breites Themenspektrum bearbeiten, befasst sich die FHDGGV konkret mit hydrogeologischen Fragestellungen oder mit Themen, die damit direkt im Zusammenhang stehen. Daraus resultieren zwangsläufig fachliche Überschneidungen, die im Jahre 2003 zu einer Kooperationsvereinbarung mit dem DVGW und der DWA geführt haben. Durch diese Kooperation sollen Synergien gestärkt und Doppelarbeit vermieden werden. Darüber hinaus sollen die Mitglieder der beteiligten Verbände insbesondere in Bezug auf das Grundwasser zusätzliche Informationen über Bildungsveranstaltungen, Publikationen u. ä. erhalten. Auch wurden Vertreter der FH-DGGV in diverse Gremien von DVGW und DWA entsandt. Umgekehrt stellen DWA und DVGW jeweils einen Vertreter, welcher als Gast an den Sitzungen des Vorstands und Beirats der FH-DGGV teilnimmt. Diese erfolgreiche Kooperation hat bis heute Bestand.

In der vergangenen Dekade war die FH-DGGV im Rahmen der Kooperation an zahlreichen Vorhaben beteiligt. Besonders wäre hier die Mitarbeit an der DVGW-Information Wasser Nr. 83 ,Unterirdische Nutzungen und Gewässerschutz“ zu nennen, die sich im Hinblick auf den Grundwasserschutz mit neuen Gefahrenquellen für die Wasserversorgung auseinandersetzt. Auch ist geplant, mit Beteiligung der FH-DGGV das DVGW-Arbeitsblatt W 101 (Richtlinien für Trinkwasserschutzgebiete) zu überarbeiten. Gleiches ist bereits mit dem DVGW-Arbeitsblatt W 107 (Aufbau und Anwendung numerischer Grundwassermodelle in Wassergewinnungsgebieten) geschehen.

Mit einem Rückblick auf die vergangenen 12 Jahre kann resümiert werden, dass sich die Kooperation der Fachsektion Hydrogeologie mit dem DVGW und der DWA bewährt hat. 